\title{
A FUJITA-TYPE BLOWUP RESULT AND LOW ENERGY SCATTERING FOR A NONLINEAR SCHRÖDINGER EQUATION
}

\author{
THIERRY CAZENAVE ${ }^{1}$, SIMÃO CORREIA ${ }^{2}$, FLÁVIO DICKSTEIN $^{3}$, \\ AND FRED B. WEISSLER ${ }^{4}$
}

\begin{abstract}
In this paper we consider the nonlinear Schrödinger equation $i u_{t}+$ $\Delta u+\kappa|u|^{\alpha} u=0$. We prove that if $\alpha<\frac{2}{N}$ and $\Im \kappa<0$, then every nontrivial $H^{1}$-solution blows up in finite or infinite time. In the case $\alpha>\frac{2}{N}$ and $\kappa \in \mathbb{C}$, we improve the existing low energy scattering results in dimensions $N \geq 7$. More precisely, we prove that if $\frac{8}{N+\sqrt{N^{2}+16 N}}<\alpha \leq \frac{4}{N}$, then small data give rise to global, scattering solutions in $H^{1}$.
\end{abstract}

\section{INTRODUCTION}

The main purpose of this article is to prove a Fujita-type blowup result for the nonlinear Schrödinger equation

$$
i u_{t}+\Delta u+\kappa|u|^{\alpha} u=0 .
$$

Given an initial value $u_{0}$, the Cauchy problem for (1.1) has the equivalent form

$$
u(t)=e^{i t \Delta} u_{0}+i \kappa \int_{0}^{t} e^{i(t-s) \Delta}\left(|u|^{\alpha} u\right)(s) d s .
$$

As is well known, the Cauchy problem (1.2) is locally well-posed in $H^{1}\left(\mathbb{R}^{N}\right)$ provided $\alpha<\frac{4}{N-2}$. (See [10].) More precisely, given $u_{0} \in H^{1}\left(\mathbb{R}^{N}\right)$, there exist a maximal existence time $T_{\max }=T_{\max }\left(u_{0}\right) \in(0, \infty]$ and a unique solution $u \in C\left(\left[0, T_{\max }\right), H^{1}\left(\mathbb{R}^{N}\right)\right)$ of (1.2). Moreover, if $T_{\max }<\infty$, then $u$ blows up at $T_{\max }$ in the sense that $\|u(t)\|_{H^{1}} \rightarrow \infty$ as $t \uparrow T_{\max }$.

Recall that Fujita [7] proved that if $\alpha<\frac{2}{N}$, then all positive solutions of the nonlinear heat equation

$$
u_{t}=\Delta u+|u|^{\alpha} u
$$

on $\mathbb{R}^{N}$ blow up in finite time. In addition, if $\alpha>\frac{2}{N}$, then for initial values sufficiently small in an appropriate sense, the corresponding solution of (1.3) is global in time. See [7]. In the intervening years, this classical result has lead to an extensive literature, see the two survey articles $[12,5]$. However, the extensions have always been to parabolic equations.

2010 Mathematics Subject Classification. Primary: 35Q55. Secondary: 35Q56, 35B33, 35B40, 35B44.

Key words and phrases. Nonlinear Schrödinger equation, Fujita critical exponent, low energy scattering.

Research supported by the "Brazilian-French Network in Mathematics".

Flávio Dickstein was partially supported by CNPq (Brasil), and by the Fondation Sciences Mathématiques de Paris.

Simão Correia was partially supported by FCT (Portugal) through the grant SFRH/BD/96399/2013. 
It turns out that there is a similar blowup dichotomy for the nonlinear Schrödinger equation (1.1). The blowup part of this dichotomy concerns the case $\Im \kappa<0$. Indeed, if $\kappa \in \mathbb{R}$, in which case (1.1) becomes the standard nonlinear Schrödinger equation, well-known energy estimates imply that if $\alpha<\frac{4}{N}$, then all $H^{1}$-solutions are global in time and remain bounded. These arguments yield the same result if $\Im \kappa>0$. On the other hand, we prove that if $\varsigma \kappa<0$ and $\alpha<\frac{2}{N}$, there is no global, nontrivial solution of (1.1) that remains bounded in $H^{1}\left(\mathbb{R}^{N}\right)$. More precisely, we prove the following result.

Theorem 1.1. Assume $\Im \kappa<0$ and $\alpha<\frac{2}{N}$. It follows that there exists $\delta>0$ such that if $u \neq \equiv 0$ is a global $H^{1}$ solution of (1.1), then

$$
\sup _{0 \leq s \leq t}\|\nabla u(s)\|_{L^{2}} \geq \delta\|u(0)\|_{L^{2}}^{\frac{N+2}{N}} t^{\frac{2-N \alpha}{N \alpha}}
$$

for all $t>0$. Moreover,

$$
t^{-\frac{2-N \alpha}{N \alpha}} \sup _{0 \leq s \leq t}\|\nabla u(s)\|_{L^{2}} \underset{t \rightarrow \infty}{\longrightarrow} \infty .
$$

In other words, every nontrivial $H^{1}$-solution blows up in finite or infinite time. We expect that blowup in fact occurs in finite time.

Concerning the global existence part of the dichotomy, it is natural to conjecture that if $\alpha>\frac{2}{N}$, then initial values which are sufficiently small in some norm lead to global solutions which remain bounded and have scattering states in $H^{1}$. This is in fact known in dimension $N=1,2,3$. (See [4, 8, 13].) In higher dimension $N \geq 4$, the best available result seems to be global existence and scattering for small data (i.e. low energy scattering) when $N \alpha+2 \alpha+2 \alpha^{2}>4$, i.e. $\alpha>\alpha_{1}$ where

$$
\alpha_{1}=\frac{8}{N+2+\sqrt{N^{2}+4 N+36}}
$$

(See $[8,13]$.

The contribution of this paper to the case $\alpha>\frac{2}{N}$ is that we improve the condition $\alpha>\alpha_{1}$ when $N \geq 7$, to $\alpha>\alpha_{2}$ with

$$
\alpha_{2}=\frac{8}{N+\sqrt{N^{2}+16 N}}
$$

Our result in this case is the following.

Theorem 1.2. Set $X=H^{1}\left(\mathbb{R}^{N}\right) \cap L^{2}\left(\mathbb{R}^{N},|x|^{2} d x\right)$ equipped with its natural norm. Let $\kappa \in \mathbb{C}$ and assume

$$
N \geq 3, \quad \alpha_{2}<\alpha<\frac{4}{N}
$$

where $\alpha_{2}$ is given by (1.7). Let $u_{0} \in X$ satisfy $v_{0} \in H^{2}\left(\mathbb{R}^{N}\right)$ and $|\cdot| v_{0} \in H^{1}\left(\mathbb{R}^{N}\right)$, where $v_{0}(x)=e^{i \frac{|x|^{2}}{4}} u_{0}(x)$. If $\left\|v_{0}\right\|_{H^{2}}$ is sufficiently small, then the solution of (1.2) is global. Moreover, there exists $u^{+} \in X$ such that $e^{-i t \Delta} u(t) \rightarrow u^{+}$in $X$ as $t \rightarrow \infty$.

Note that $\alpha_{2}<\frac{4}{N}$ and behaves as $\frac{4}{N}$ as $N \rightarrow \infty$, not as $\frac{2}{N}$. Thus there is still a significant gap in high dimensions between the conjecture and the known results.

A fundamental technical tool used in the proofs of the above cited results $[4,8,13]$ is the Strichartz inequalities. These inequalities involve space-time integrals, where the pair of Lebesgue indices satisfy a certain relationship. Usually, the pairs of Lebesgue indices are admissible (see [3, p. 808]), and in particular the low energy 
scattering results of $[4,8,13]$ use admissible pairs. Strichartz estimates with nonadmissible pairs first appeared in [4, Lemma 2.1], but were not used there for low energy scattering. They have subsequently been developed in $[11,6,16]$. In this paper, we use Strichartz estimates with non-admissible pairs along with the low energy scattering argument of [4]. This combination enables us to prove low energy scattering for $\alpha>\alpha_{2}$.

It is worth noting that the exponent $\alpha=\frac{2}{N}$ is also the critical exponent related to scattering of solutions of (1.1). When $\alpha<\frac{2}{N}$ in dimension $N \geq 2$, it is known that no nonzero solution of (1.1) can be global and scatter in $L^{2}\left(\mathbb{R}^{N}\right)$. (See Strauss [15], Theorem 3.2 and Example 3.3, p. 68.)

Theorems 1.1 and 1.2 are proved respectively in Sections 2 and 3 below.

\section{BLOWUP}

The remarkable feature of (1.1) is the identity

$$
\frac{1}{2} \frac{d}{d t} \int_{\mathbb{R}^{N}}|u|^{2}=-\Im \kappa \int_{\mathbb{R}^{N}}|u|^{\alpha+2}
$$

which holds for all $0 \leq t<T_{\max }$. (When $\Im \kappa=0$, this is the conservation of charge for the standard NLS.) We observe that, were the equation set on a bounded domain with Dirichlet boundary conditions, equation (2.1) together with Hölder's inequality would imply that no $H^{1}$ solution can be global (for all $\alpha>0$ ), when $\Im \kappa<0$.

Proof of Theorem 1.1. Let $u(t) \not \equiv 0$ be a global $H^{1}$ solution of (1.1). The idea of the proof is to multiply equation (1.1) by a cut-off function, so that the $L^{2}$ norm can be controlled by the $L^{\alpha+2}$ norm. We fix the cut-off function $\psi(x)=\nu \theta(|x|)$, where

$$
\theta(r)= \begin{cases}1 & 0 \leq r \leq 1 \\ 2-r & 1 \leq r \leq 2 \\ 0 & r \geq 2\end{cases}
$$

and $\nu \in \mathbb{R}$ is such $\|\psi\|_{L^{2}}=1$. Given $\lambda>0$, set

$$
\varphi_{\lambda}(x)=\psi(\lambda x) .
$$

It follows in particular that $\varphi_{\lambda} \in C_{\mathrm{c}}\left(\mathbb{R}^{N}\right) \cap W^{1, \infty}\left(\mathbb{R}^{N}\right), \varphi_{\lambda} \geq 0$,

$$
\left\|\varphi_{\lambda}\right\|_{L^{2}}=\lambda^{-\frac{N}{2}} \text { and }\left\|\nabla \varphi_{\lambda}\right\|_{L^{\infty}}=\nu \lambda .
$$

Multiplying equation (1.1) by $\varphi_{\lambda}^{2} \bar{u}$ and taking the imaginary part, we obtain

$$
\frac{1}{2} \frac{d}{d t} \int_{\mathbb{R}^{N}}|u|^{2} \varphi_{\lambda}^{2}=2 \Im \int_{\mathbb{R}^{N}} \bar{u} \varphi_{\lambda} \nabla u \cdot \nabla \varphi_{\lambda}-\Im \kappa \int_{\mathbb{R}^{N}}|u|^{\alpha+2} \varphi_{\lambda}^{2} .
$$

(To be precise, the equation makes sense in $H^{-1}$, so we take the $H^{-1}-H^{1}$ duality bracket of the equation with $\varphi_{\lambda}^{2} \bar{u} \in H^{1}$.) Set

$$
f_{\lambda}(t)=\left\|u \varphi_{\lambda}\right\|_{L^{2}}
$$

and

$$
K_{t}=\|\nabla u\|_{L^{\infty}\left((0, t), L^{2}\right)} .
$$

It follows from Hölder's inequality and (2.4) that

$$
f_{\lambda}(t)^{\alpha+2} \leq\left\|\varphi_{\lambda}\right\|_{L^{2}}^{\alpha} \int_{\mathbb{R}^{N}}|u|^{\alpha+2} \varphi_{\lambda}^{2}=\lambda^{-\frac{N \alpha}{2}} \int_{\mathbb{R}^{N}}|u|^{\alpha+2} \varphi_{\lambda}^{2},
$$


so that

$$
\int_{\mathbb{R}^{N}}|u|^{\alpha+2} \varphi_{\lambda}^{2} \geq \lambda^{\frac{N \alpha}{2}} f_{\lambda}(t)^{\alpha+2}
$$

Moreover, we deduce from Hölder's inequality, (2.4), (2.6) and (2.7) that

$$
\left|\Im \int_{\mathbb{R}^{N}} \bar{u} \varphi_{\lambda} \nabla u \cdot \nabla \varphi_{\lambda}\right| \leq \nu \lambda K_{t} f_{\lambda}(t) .
$$

Consequently, (2.5), (2.8) and (2.9) yield

$$
f_{\lambda}^{\prime} \geq-2 \nu \lambda K_{t}-\Im \kappa \lambda^{\frac{N \alpha}{2}} f_{\lambda}^{\alpha+1} \geq-2 \nu \lambda K_{T}-\Im \kappa \lambda^{\frac{N \alpha}{2}} f_{\lambda}^{\alpha+1}
$$

for all $0<t \leq T<\infty$, where we used the property that $K_{t}$ is nondecreasing in $t$ in the last inequality. Therefore, if

$$
f_{\lambda}(0)^{\alpha+1} \geq 4(-\Im \kappa)^{-1} \lambda^{\frac{2-N \alpha}{2}} \nu K_{T},
$$

it follows that $f_{\lambda}$ is increasing on $(0, T)$, and

$$
f_{\lambda}^{\prime} \geq \frac{-\Im \kappa}{2} \lambda^{\frac{N \alpha}{2}} f_{\lambda}^{\alpha+1}
$$

on $(0, T)$. Equation (2.12) implies that $f_{\lambda}$ must blow up before the finite time $\frac{2}{-\Im \kappa \alpha} \lambda^{-\frac{N \alpha}{2}} f_{\lambda}(0)^{-\alpha}$. Therefore,

$$
T \leq \frac{2}{-\Im \kappa \alpha} \lambda^{-\frac{N \alpha}{2}} f_{\lambda}(0)^{-\alpha} .
$$

Note that $f_{\lambda}(0)$ is a nonincreasing function of $\lambda>0$ and

$$
f_{\lambda}(0)=\left(\int_{\mathbb{R}^{N}}|u(0, x)|^{2} \psi^{2}(\lambda x) d x\right)^{\frac{1}{2}} \longrightarrow \begin{cases}0 & \text { as } \lambda \uparrow \infty \\ \|u(0, \cdot)\|_{L^{2}} & \text { as } \lambda \downarrow 0 .\end{cases}
$$

We first show that

$$
K_{T} \underset{T \rightarrow \infty}{\longrightarrow} \infty .
$$

Indeed, suppose by contradiction that $K_{T}$ is bounded as $T \rightarrow \infty$. It follows from (2.14) that we can choose $\widetilde{\lambda}>0$ sufficiently small so that (2.11) with $\lambda=\widetilde{\lambda}$ holds for all $T>0$. We deduce from $(2.13)$ that $T \leq \frac{2}{-\Im \kappa \alpha} \widetilde{\lambda}^{-\frac{N \alpha}{2}} f_{\widetilde{\lambda}}(0)^{-\alpha}$ for all $T>0$. This is absurd, proving (2.15).

We next prove (1.4). Fix $\lambda_{0}>0$ such that

$$
f_{\lambda_{0}}(0)=\frac{1}{2}\|u(0, \cdot)\|_{L^{2}}
$$

Note that this is possible by (2.14). It follows from (2.15) that if $T>0$ is sufficiently large, $\lambda=\lambda(T)$ defined by

$$
f_{\lambda_{0}}(0)^{\alpha+1}=4(-\Im \kappa)^{-1} \lambda(T)^{\frac{2-N \alpha}{2}} K_{T},
$$

satisfies

$$
\lambda(T) \leq \lambda_{0} .
$$

Since $f_{\lambda}(0)$ is a nonincreasing function of $\lambda$, we deduce from (2.17)-(2.18) that (2.11) holds with $\lambda=\lambda(T)$. Therefore, it follows from (2.13) that

$$
T \leq \frac{2}{-\Im \kappa \alpha} \lambda(T)^{-\frac{N \alpha}{2}} f_{\lambda(T)}(0)^{-\alpha} .
$$


Using again (2.18), we deduce from (2.19) that

$$
T \leq \frac{2}{-\Im \kappa \alpha} \lambda(T)^{-\frac{N \alpha}{2}} f_{\lambda_{0}}(0)^{-\alpha}
$$

Since (2.17) implies

$$
\lambda(T)^{-\frac{2-N \alpha}{2}}=\frac{4}{-\Im \kappa} K_{T} f_{\lambda_{0}}(0)^{-(\alpha+1)}
$$

formulas (2.20) and (2.21) yield

$$
\begin{aligned}
T^{\frac{2-N \alpha}{N \alpha}} & \leq \frac{4}{-\Im \kappa}\left(\frac{2}{-\Im \kappa \alpha} f_{\lambda_{0}}(0)^{-\alpha}\right)^{\frac{2-N \alpha}{N \alpha}} f_{\lambda_{0}}(0)^{-(\alpha+1)} K_{T} \\
& =2^{\frac{2+N \alpha}{N \alpha}}(-\Im \kappa)^{-\frac{2}{N \alpha}} \alpha^{-\frac{2-N \alpha}{N \alpha}} f_{\lambda_{0}}(0)^{-\frac{N+2}{N}} K_{T} .
\end{aligned}
$$

Inequality (1.4) now follows from (2.22) and (2.16).

We finally prove (1.5). Given $T>0$, it follows from (2.14) that there exists a unique $\mu(T)>0$ such that

$$
f_{\mu(T)}(0)^{\alpha+1}=4(-\Im \kappa)^{-1} \mu(T)^{\frac{2-N \alpha}{2}} K_{T} .
$$

Since $K_{T}$ is a nondecreasing function of $T$, it follows from (2.23) that the map $T \mapsto f_{\mu(T)}(0)^{\alpha+1} \mu(T)^{-\frac{2-N \alpha}{2}}$ is also nondecreasing. On the other hand, $f_{\mu}(0)$ is a nonincreasing function of $\mu$, so that the map $\mu \mapsto f_{\mu}(0)^{\alpha+1} \mu^{-\frac{2-N \alpha}{2}}$ is decreasing, so we conclude that the map $T \mapsto \mu(T)$ is nonincreasing. Since $f_{\mu}(0) \leq\|u(0)\|_{L^{2}}$ for all $\mu>0$ by (2.14) and $K_{T} \geq \delta\|u(0)\|_{L^{\frac{N+2}{N}}}^{\frac{N-N \alpha}{N \alpha}}$ by (1.4) we deduce from (2.23) that

$$
\mu(T) \leq\left(4(-\Im \kappa)^{-1} \delta\right)^{-\frac{2}{2-N \alpha}}\|u(0)\|_{L^{2}}^{-\frac{2}{N}} T^{-\frac{2}{N \alpha}} \underset{T \rightarrow \infty}{\longrightarrow} 0 .
$$

We deduce in particular from (2.14) and (2.24) that $f_{\mu(T)}(0) \rightarrow\|u(0)\|_{L^{2}}$ as $T \rightarrow \infty$ so that by $(2.23)$

$$
\mu(T)^{\frac{2-N \alpha}{2}} K_{T} \underset{T \rightarrow \infty}{\longrightarrow} \frac{\|u(0)\|_{L^{2}}^{\alpha+1}(-\Im \kappa)}{4} .
$$

Moreover, it follows from (2.23) that (2.11) is satisfied with $\lambda=\mu(T)$, so that (2.12) holds, i.e.

$$
f_{\mu(T)}^{\prime} \geq \frac{-\Im \kappa}{2} \mu(T)^{\frac{N \alpha}{2}} f_{\mu(T)}^{\alpha+1}
$$

for all $0<t<T$. Integrating the above differential inequality on $(T / 2, T)$ and using (2.25), we obtain

$$
f_{\mu(T)}(T / 2)^{-\alpha}-f_{\mu(T)}(T)^{-\alpha} \geq \frac{-\Im \kappa \alpha}{4} \mu(T)^{\frac{N \alpha}{2}} T \geq \eta K_{T}^{-\frac{N \alpha}{2-N \alpha}} T
$$

for $T \geq 2$, with $\eta>0$. Next, recall that $\mu(t)$ is a nonincreasing function of $t$, and that the map $\lambda \mapsto f_{\lambda}(t)$ is a nonincreasing function of $\lambda$, so that

$$
f_{\mu(s)}(\tau) \leq f_{\mu(t)}(\tau)
$$

for all $\tau>0$ and $0<s<t$. Therefore, letting $s=\tau=T / 2$ and $t=T$, we see that $f_{\mu(T / 2)}(T / 2)^{-\alpha} \geq f_{\mu(T)}(T / 2)^{-\alpha}$ and it follows from (2.27) that

$$
f_{\mu(T / 2)}(T / 2)^{-\alpha}-f_{\mu(T)}(T)^{-\alpha} \geq \eta K_{T}^{-\frac{N \alpha}{2-N \alpha}} T
$$


for $T \geq 2$. Next, we deduce from (2.28) and the fact that the map $\tau \mapsto f_{\mu(t)}(\tau)$ is increasing on $(0, t)$, that

$$
f_{\mu(s)}(s) \leq f_{\mu(t)}(s) \leq f_{\mu(t)}(t)
$$

for all $0<s<t$. Thus we see that the map $t \mapsto f_{\mu(t)}(t)$ is nondecreasing; and so the map $t \mapsto f_{\mu(t)}(t)^{-\alpha}$ is nonincreasing, so it has a limit as $t \rightarrow \infty$. Letting $T \rightarrow \infty$ in (2.29), we deduce that $K_{T}^{-\frac{N \alpha}{2-N \alpha}} T \rightarrow 0$ as $T \rightarrow \infty$, which is the desired result.

Remark 2.1. Under the assumption

$$
-\Im \kappa \geq \frac{\alpha}{2 \sqrt{\alpha+1}}|\Re \kappa|
$$

we may replace $\sup _{0 \leq s \leq t}\|\nabla u(s)\|_{L^{2}}$ by $\|\nabla u(t)\|_{L^{2}}$ in estimates (1.4) and (1.5) of Theorem 1.1. Indeed, it follows from (2.31) that $\|\nabla u(t)\|_{L^{2}}$ is a nondecreasing function of $t$. To see this, note that for a solution of (1.1) we have

$$
\frac{1}{2} \frac{d}{d t}\|\nabla u(t)\|_{L^{2}}^{2}=\Re\left(i \kappa \int_{\mathbb{R}^{N}} \nabla\left(|u|^{\alpha} u\right) \cdot \nabla \bar{u}\right) \stackrel{\text { def }}{=} A .
$$

Since

$$
\nabla\left(|u|^{\alpha} u\right)=\frac{\alpha+2}{2}|u|^{\alpha} \nabla u+\frac{\alpha}{2}|u|^{\alpha-2} u^{2} \nabla \bar{u}
$$

we see that

$$
\nabla\left(|u|^{\alpha} u\right) \cdot \nabla \bar{u}=\frac{\alpha+2}{2}|u|^{\alpha}|\nabla u|^{2}+\frac{\alpha}{2}|u|^{\alpha-2} u^{2}(\nabla \bar{u})^{2} .
$$

It follows that

$$
\begin{aligned}
\Re\left[i \kappa \nabla\left(|u|^{\alpha} u\right) \cdot \nabla \bar{u}\right] & =-\Im \kappa \frac{\alpha+2}{2}|u|^{\alpha}|\nabla u|^{2}+\frac{\alpha}{2} \Re\left[i \kappa|u|^{\alpha-2} u^{2}(\nabla \bar{u})^{2}\right] \\
& \geq\left(-\frac{\alpha+2}{2} \Im \kappa-\frac{\alpha}{2}|\kappa|\right)|u|^{\alpha}|\nabla u|^{2} .
\end{aligned}
$$

This shows that $A \geq 0$ provided (2.31) holds. The above calculations are justified if $u$ is an $H^{2}$ solution. The result follows by approximation, regularity, and continuous dependence. (All these properties are established in [10].) Note that (2.31) is identical to condition (2.2) in [14].

Remark 2.2. Under the assumptions of Theorem 1.1, we do not know whether or not there exists a global $H^{1}$ solution of (1.1). In fact, if such a solution does exist, it would necessarily have a stronger dispersion than the solutions of the linear Schrödinger equation. Indeed, suppose $u \not \equiv 0$ is a global $H^{1}$ solution of (1.1) and let $R(t)$ satisfy

We claim that

$$
\int_{\{|x| \leq R(t)\}}|u(t, x)|^{2} d x=\frac{1}{2} \int_{\mathbb{R}^{N}}|u(t, x)|^{2} d x .
$$

$$
\limsup _{t \rightarrow \infty} t^{-\frac{2}{N \alpha}} R(t)=\infty .
$$

To see this, observe that by Hölder's inequality and the definition of $R(t)$,

$$
\int_{\mathbb{R}^{N}}|u|^{\alpha+2} \geq 2^{-\frac{\alpha+2}{2}} \omega_{N}^{-\frac{\alpha}{2}} R^{-\frac{N \alpha}{2}} f(t)^{\frac{\alpha+2}{2}}
$$


where $f(t)=\int_{\mathbb{R}^{N}}|u|^{2}$ and $\omega_{N}$ is the measure of the unit ball of $\mathbb{R}^{N}$. It follows from (1.1) and (2.37) that

$$
f^{\prime} \geq-\Im \kappa 2^{-\frac{\alpha}{2}} \omega_{N}^{-\frac{\alpha}{2}} R^{-\frac{N \alpha}{2}} f^{\frac{\alpha+2}{2}} .
$$

Therefore, $\int_{0}^{\infty} R(t)^{-\frac{N \alpha}{2}} d t<\infty$, which yields (2.36). On the other hand, let $\widetilde{u}(t)=$ $e^{i t \Delta} u_{0}$ where $u_{0} \in H^{1}\left(\mathbb{R}^{N}\right), u_{0} \neq 0$. Multiplying the equation $i \widetilde{u}_{t}+\Delta \widetilde{u}=0$ by $\psi_{M} \bar{u}$, where $\psi_{M}(x)=\min \left\{\frac{x}{M}, 1\right\}$, we obtain

$$
\int_{\mathbb{R}^{N}} \psi_{M}|\widetilde{u}|^{2} \leq \int_{\mathbb{R}^{N}} \psi_{M}\left|u_{0}\right|^{2}+\frac{2 t}{M}\left\|u_{0}\right\|_{L^{2}}\left\|\nabla u_{0}\right\|_{L^{2}}
$$

(Cf. [9, Lemma 5.4]).) Given $t>0$, we substitute $M=a t$ in (2.38) with $a=$ $16 \frac{\left\|\nabla u_{0}\right\|_{L^{2}}}{\left\|u_{0}\right\|_{L^{2}}}$. Since $\psi_{M} \geq 1_{\{|x|>M\}}$, this yields

$$
\int_{\{|x|>a t\}}|\widetilde{u}|^{2} \leq \int_{\mathbb{R}^{N}} \psi_{a t}\left|u_{0}\right|^{2}+\frac{1}{8}\left\|u_{0}\right\|_{L^{2}}^{2} .
$$

Furthermore, $\int_{\mathbb{R}^{N}} \psi_{a t}\left|u_{0}\right|^{2} \rightarrow 0$ as $t \rightarrow \infty$ by dominated convergence, and so

$$
\int_{\{|x|>a t\}}|\widetilde{u}|^{2} \leq \frac{1}{4}\left\|u_{0}\right\|_{L^{2}}^{2}
$$

for $t$ large. Therefore,

$$
\int_{\{|\widetilde{x}|<a t\}}|u|^{2} \geq \frac{3}{4}\left\|u_{0}\right\|_{L^{2}}^{2}
$$

for $t$ large. In particular, if $\widetilde{R}(t)$ satisfies

$$
\int_{\{|x| \leq \widetilde{R}(t)\}}|\widetilde{u}(t, x)|^{2} d x=\frac{1}{2} \int_{\mathbb{R}^{N}}|\widetilde{u}(t, x)|^{2} d x=\frac{1}{2}\left\|u_{0}\right\|_{L^{2}}^{2},
$$

then $\widetilde{R}(t) \leq a t$ for $t$ large. Comparing with (2.36), we see that $u$ has a stronger dispersion than $\widetilde{u}$ as $t \rightarrow \infty$.

Remark 2.3. If we look for solutions of (1.1) of the form

$$
u(t, x)=\rho(t) e^{i \frac{|x|^{2}}{4\left(t+t_{0}\right)}},
$$

where $t_{0}>0$ is given, then $\rho$ must satisfy

$$
\rho^{\prime}=-\frac{N}{2\left(t+t_{0}\right)} \rho-i \kappa|\rho|^{\alpha} \rho .
$$

Setting $z=\left(t+t_{0}\right)^{\frac{N}{2}} \rho$, we get to the equation

$$
z^{\prime}=-i \kappa\left(t+t_{0}\right)^{-\frac{N \alpha}{2}}|z|^{\alpha} z .
$$

Multiplying the equation by $\bar{z}$ and taking the real part, one easily gets to

$$
\frac{1}{\alpha|z(t)|^{\alpha}}=\frac{1}{\alpha|z(0)|^{\alpha}}+\Im \kappa \int_{0}^{t} \frac{d s}{\left(s+t_{0}\right)^{\frac{N \alpha}{2}}} .
$$

If $\alpha>\frac{2}{N}$, then the integral on the right-hand side of (2.40) is convergent, and we see that if $|z(0)|$ is sufficiently small so that

$$
\frac{1}{\alpha|z(0)|^{\alpha}} \geq-\Im \kappa \int_{0}^{\infty} \frac{d s}{\left(s+t_{0}\right)^{\frac{N \alpha}{2}}}
$$


then the solution is global; and if $|z(0)|$ is larger, then the solution blows up in finite time. On the other hand, if $\alpha \leq \frac{2}{N}$, then the integral on the right-hand side of (2.40) is divergent. Therefore, for every $z(0)$, the solution blows up at the finite time $T$ given by

$$
\frac{1}{\alpha|z(0)|^{\alpha}}=-\Im \kappa \int_{0}^{T} \frac{d s}{\left(s+t_{0}\right)^{\frac{N \alpha}{2}}} .
$$

Thus we see that the exponent $\alpha=\frac{2}{N}$ is critical.

\section{LOW ENERGY SCATTERING}

To prove Theorem 1.2, we first prove a local existence result for small data for the following equation

$$
v(t)=e^{i t \Delta} v_{0}+\int_{0}^{t} h(s) e^{i(t-s) \Delta}\left(|v|^{\alpha} v\right)(s) d s
$$

where

$$
h(t)=i \kappa(1-t)^{-\frac{4-N \alpha}{2}}
$$

for $0 \leq t<1$. As we will see, equation (3.1) is equivalent to equation (1.1) via the pseudo-conformal transformation. Before stating the result, we introduce some notation. We assume (1.8) and we set

$$
\rho=\frac{N(\alpha+2)}{N+\alpha}, \quad \gamma=\frac{4(\alpha+2)}{\alpha(N-2)}=\frac{4 \rho}{N(\rho-2)} .
$$

It is not difficult to show that

$$
2<\rho<\frac{2 N}{N-2}, \quad N>\rho
$$

and that $(\gamma, \rho)$ is an admissible pair, i.e. $\frac{2}{\gamma}=N\left(\frac{1}{2}-\frac{1}{\rho}\right)$ (see [3, Proposition 1.5 (ii)]). Note also that $\alpha>\alpha_{2}$, which implies $N \alpha^{2}+N \alpha>4$, so that

$$
\frac{4-(N-4) \alpha}{2(\alpha+2)}>\frac{4-N \alpha}{2}
$$

Theorem 3.1. Suppose $N \geq 3, \kappa \in \mathbb{C}$ and $\alpha_{2}<\alpha<\frac{4}{N}$ where $\alpha_{2}$ is given by (1.7). Fix

$$
a \geq \gamma
$$

sufficiently large so that

$$
\frac{4-(N-4) \alpha}{2(\alpha+2)}-\frac{\alpha}{a}>\frac{4-N \alpha}{2} .
$$

(The existence of a is guaranteed by (3.5).) There exists $\delta>0$ such that if

$$
\begin{gathered}
v_{0} \in X \\
(1+|\cdot|) e^{i t \Delta} v_{0} \in L^{a}\left((0,1), L^{\rho}\left(\mathbb{R}^{N}\right)\right) \\
e^{i t \Delta} \nabla v_{0} \in L^{a}\left((0,1), L^{\rho}\left(\mathbb{R}^{N}\right)\right) \\
\left\|\nabla e^{i t \Delta} v_{0}\right\|_{L^{a}\left((0,1), L^{\rho}\right)} \leq \delta
\end{gathered}
$$

then there exists a solution $v \in C([0,1], X)$ of $(3.1)$. 
Proof of Theorem 3.1. We define $\widetilde{a}$ by

$$
\frac{1}{a}+\frac{1}{\widetilde{a}}=\frac{2}{\gamma}
$$

and we recall the following Strichartz-type estimate for non-admissible pairs

$$
\left\|\int_{0}^{\cdot} e^{i(\cdot-s) \Delta} f(s) d s\right\|_{L^{a}\left((0,1), L^{\rho}\right)} \leq C\|f\|_{L^{\tilde{a}^{\prime}}\left((0,1), L^{\rho^{\prime}}\right)} .
$$

(See [4, Lemma 2.1].) If $\mu$ is defined by

$$
\frac{1}{\mu}=\frac{4-(N-4) \alpha}{2(\alpha+2)}-\frac{\alpha}{a}<\frac{4-(N-4) \alpha}{2(\alpha+2)}<1
$$

then it follows from (3.7) and (3.2) that

$$
h \in L^{\mu}(0,1) .
$$

Next, we deduce from Sobolev's inequality that

$$
\|v\|_{L^{\frac{\alpha \rho}{\rho-2}}}=\|v\|_{L^{\frac{N \rho}{N-\rho}}} \leq C\|\nabla v\|_{L^{\rho}}
$$

and so by Hölder's inequality,

$$
\left\||v|^{\alpha} w\right\|_{L^{\rho^{\prime}}} \leq\|v\|_{L^{\frac{\alpha \rho}{\rho-2}}}^{\alpha}\|w\|_{L^{\rho}} \leq C\|\nabla v\|_{L^{\rho}}^{\alpha}\|w\|_{L^{\rho}} .
$$

It easily follows from (3.17) that

$$
\begin{gathered}
\left\||v|^{\alpha} v\right\|_{L^{\rho^{\prime}}} \leq C\|\nabla v\|_{L^{\rho}}^{\alpha}\|v\|_{L^{\rho}} \\
\left\||\cdot||v|^{\alpha} v\right\|_{L^{\rho^{\prime}}} \leq C\|\nabla v\|_{L^{\rho}}^{\alpha}\||\cdot| v\|_{L^{\rho}} \\
\left\|\nabla\left(|v|^{\alpha} v\right)\right\|_{L^{\rho^{\prime}}} \leq C\|\nabla v\|_{L^{\rho}}^{\alpha+1} \\
\left\||u|^{\alpha} u-|v|^{\alpha} v\right\|_{L^{\rho^{\prime}}} \leq C\left(\|\nabla u\|_{L^{\rho}}^{\alpha}+\|\nabla v\|_{L^{\rho}}^{\alpha}\right)\|u-v\|_{L^{\rho} .}
\end{gathered}
$$

We observe, by (3.14) and (3.12), that

$$
\frac{1}{\widetilde{a}^{\prime}}=\frac{1}{\mu}+\frac{\alpha+1}{a} \text {. }
$$

Again using Hölder's inequality, but with the time integrals, we deduce from (3.22), along with respectively (3.18), (3.19), (3.20) and (3.21), that

$$
\begin{gathered}
\left\|h|v|^{\alpha} v\right\|_{L^{\tilde{a}^{\prime}\left((0,1), L^{\rho^{\prime}}\right)}} \leq C\|h\|_{L^{\mu}(0,1)}\|\nabla v\|_{L^{a}\left((0,1), L^{\rho}\right)}^{\alpha}\|v\|_{L^{a}\left((0,1), L^{\rho}\right)} \\
\left\|h|\cdot||v|^{\alpha} v\right\|_{L^{\tilde{a}^{\prime}}\left((0,1), L^{\rho^{\prime}}\right)} \leq C\|h\|_{L^{\mu}(0,1)}\|\nabla v\|_{L^{a}\left((0,1), L^{\rho}\right)}^{\alpha}\|\cdot \mid v\|_{L^{a}\left((0,1), L^{\rho}\right)} \\
\left\|\nabla\left(h|v|^{\alpha} v\right)\right\|_{L^{\tilde{a}^{\prime}}\left((0,1), L^{\rho^{\prime}}\right)} \leq C\|h\|_{L^{\mu}(0,1)}\|\nabla v\|_{L^{a}\left((0,1), L^{\rho}\right)}^{\alpha+1}
\end{gathered}
$$

and

$$
\begin{aligned}
\left\|h\left(|u|^{\alpha} u-|v|^{\alpha} v\right)\right\|_{L^{\tilde{a}^{\prime}}\left((0,1), L^{\rho^{\prime}}\right)} \leq C\|h\|_{L^{\mu}(0,1)} \\
\times\left(\|\nabla u\|_{L^{a}\left((0,1), L^{\rho}\right)}^{\alpha}+\|\nabla v\|_{L^{a}\left((0,1), L^{\rho}\right)}^{\alpha}\right)\|u-v\|_{L^{a}\left((0,1), L^{\rho}\right)}^{\alpha} .
\end{aligned}
$$

We construct the solution $v$ of (1.2) by a contraction mapping argument in the set $\mathcal{E}_{\delta, M}$ defined for $\delta, M>0$ by

$$
\begin{aligned}
& \mathcal{E}_{\delta, M}=\left\{v \in L^{a}\left((0,1), W^{1, \rho}\left(\mathbb{R}^{N}\right)\right) ;|\cdot| v \in L^{a}\left((0,1), L^{\rho}\left(\mathbb{R}^{N}\right)\right),\right. \\
& \left.\quad\|v\|_{L^{a}\left((0,1), L^{\rho}\right)} \leq M,\||\cdot| v\|_{L^{a}\left((0,1), L^{\rho}\right)} \leq M \text { and }\|\nabla v\|_{L^{a}\left((0,1), L^{\rho}\right)} \leq \delta\right\} .
\end{aligned}
$$


We set $\mathrm{d}(v, w)=\|v-w\|_{\left.L^{a}(0,1), L^{\rho}\right)}$ so that $\left(\mathcal{E}_{\delta, M}, d\right)$ is a complete metric space. Fix $v_{0} \in X$ and, given $v \in \mathcal{E}_{\delta, M}$, let $\mathcal{I}(v)$ and $\Phi(v)$ be defined by

$$
\begin{gathered}
\mathcal{I}(v)(t)=\int_{0}^{t} h(s) e^{i(t-s) \Delta}\left(|v|^{\alpha} v\right)(s) d s \\
\Phi(v)(t)=e^{i t \Delta} v_{0}+\mathcal{I}(v)(t) .
\end{gathered}
$$

It follows from (3.23), (3.25), (3.26) and the estimate (3.13) that, for some constant $C$ independent of $\delta, M$ and $v, w \in \mathcal{E}_{\delta, M}$,

$$
\begin{gathered}
\|\mathcal{I}(v)\|_{L^{a}\left((0,1), L^{\rho}\right)} \leq C\|h\|_{L^{\mu}(0,1)} \delta^{\alpha} M \\
\|\nabla \mathcal{I}(v)\|_{L^{a}\left((0,1), L^{\rho}\right)} \leq C\|h\|_{L^{\mu}(0,1)} \delta^{\alpha+1} \\
\|\mathcal{I}(v)-\mathcal{I}(w)\|_{L^{a}\left((0,1), L^{\rho}\right)} \leq C\|h\|_{L^{\mu}(0,1)} \delta^{\alpha} \mathrm{d}(v, w) .
\end{gathered}
$$

Next, we estimate the weighted norm. We observe that

$$
x e^{i \tau \Delta}=e^{i \tau \Delta}(x-2 i \tau \nabla)
$$

for all $\tau \in \mathbb{R}$. Therefore

$$
\begin{aligned}
x \mathcal{I}(v)(t) & =\int_{0}^{t} h(s) e^{i(t-s) \Delta}(x-2 i(t-s) \nabla)|v|^{\alpha} v \\
& =\int_{0}^{t} h(s) e^{i(t-s) \Delta} x|v|^{\alpha} v-2 i \int_{0}^{t} h(s)(t-s) e^{i(t-s) \Delta} \nabla\left(|v|^{\alpha} v\right)
\end{aligned}
$$

and we deduce from (3.24), (3.25) and (3.13) that

$$
\||\cdot| \mathcal{I}(v)\|_{L^{a}\left((0,1), L^{\rho}\right)} \leq C\|h\|_{L^{\mu}(0,1)} \delta^{\alpha} M .
$$

We now set

$$
\begin{gathered}
M=2 \max \left\{\left\|e^{i t \Delta} v_{0}\right\|_{L^{a}\left((0,1), L^{\rho}\right)},\left\||\cdot| e^{i t \Delta} v_{0}\right\|_{L^{a}\left((0,1), L^{\rho}\right)}\right\} \\
\delta=2\left\|\nabla v_{0}\right\|_{L^{a}\left((0,1), L^{\rho}\right)} .
\end{gathered}
$$

It follows from (3.30), (3.35) and (3.31) that if $\delta$ is sufficiently small, then

$$
\|\mathcal{I}(v)\|_{L^{a}\left((0,1), L^{\rho}\right)} \leq \frac{M}{2}, \quad\||\cdot| \mathcal{I}(v)\|_{L^{a}\left((0,1), L^{\rho}\right)} \leq \frac{M}{2}, \quad\|\nabla \mathcal{I}(v)\|_{L^{a}\left((0,1), L^{\rho}\right)} \leq \frac{\delta}{2} .
$$

Applying (3.36)-(3.37) and (3.28)-(3.29), we deduce that $\Phi: \mathcal{E}_{\delta, M} \rightarrow \mathcal{E}_{\delta, M}$. Moreover, assuming $\delta$ possibly smaller, it follows from (3.32) that $\Phi$ is a strict contraction on $\mathcal{E}_{\delta, M}$. By Banach's fixed point theorem, $\Phi$ has a fixed point $v \in \mathcal{E}_{\delta, M}$, which is a solution of (1.1).

To complete the proof, it remains to show that $v \in C([0,1], X)$. For this, we observe that by (3.6) and (3.12) we have $\widetilde{a} \leq \gamma$, so that $\widetilde{a}^{\prime} \geq \gamma^{\prime}$. Therefore, estimates (3.23), (3.24) and (3.25), and the fact that $v \in \mathcal{E}_{\delta, M}$ imply that

$$
\begin{gathered}
\left\|h|v|^{\alpha} v\right\|_{L^{\gamma^{\prime}\left((0,1), L^{\rho^{\prime}}\right.}} \leq C \delta^{\alpha} M \\
\left\|h|\cdot||v|^{\alpha} v\right\|_{L^{\gamma^{\prime}\left((0,1), L^{\rho^{\prime}}\right)}} \leq C \delta^{\alpha} M \\
\left\|\nabla\left(h|v|^{\alpha} v\right)\right\|_{L^{\gamma^{\prime}\left((0,1), L^{\rho^{\prime}}\right)}} \leq C \delta^{\alpha+1}
\end{gathered}
$$

It now follows from the standard Strichartz estimates (i.e., with admissible pairs, see e.g. [2, Theorem 2.2.3 (ii)]) that $\mathcal{I}(v) \in C([0,1], X)$. Since $v=\mathcal{I}(v)+e^{i t \Delta} v_{0}$ and $v_{0} \in X$, this completes the proof. 
Remark 3.2. The conditions (3.8)-(3.11) are satisfied under some stronger, but more familiar, conditions. Indeed, set

$$
s=N\left(\frac{1}{2}-\frac{1}{\rho}\right)-\frac{2}{a}
$$

so that $0 \leq s<1$ by (3.6) and (3.4). Setting $\frac{1}{\widetilde{\rho}}=\frac{1}{\rho}+\frac{s}{N}$, we see that $(a, \widetilde{\rho})$ is an admissible pair, so that by Strichartz's estimates (see e.g. [3, Theorem 2.2 (i)]) $\left\|e^{i t \Delta} \varphi\right\|_{L^{a}\left(\mathbb{R}, \dot{H}^{s, \tilde{\rho}}\right)} \leq C\|\varphi\|_{\dot{H}^{s}}$, where $\dot{H}^{s, p}$ and $\dot{H}^{s}=\dot{H}^{s, 2}$ are the homogeneous Sobolev spaces (see e.g. [1, Section 6.3]). Using Sobolev's embedding, we deduce that

$$
\left\|e^{i t \Delta} \varphi\right\|_{L^{a}\left(\mathbb{R}, L^{\rho}\right)} \leq C\|\varphi\|_{\dot{H}^{s}} .
$$

Therefore, conditions (3.8)-(3.11) are satisfied provided $v_{0} \in X, v_{0} \in \dot{H}^{s}\left(\mathbb{R}^{N}\right)$, $|\cdot| v_{0} \in \dot{H}^{s}\left(\mathbb{R}^{N}\right), \nabla v_{0} \in \dot{H}^{s}\left(\mathbb{R}^{N}\right)$, and the smallness condition is on the norm $\left\|\nabla v_{0}\right\|_{\dot{H}^{s}}$.

Proof of Theorem 1.2. Let $u_{0}$ be as in Theorem 1.2. In particular, $v_{0}$ defined by $v_{0}(x)=e^{i \frac{|x|^{2}}{4}} u_{0}(x)$ satisfies $v_{0} \in X$. Moreover, if $s$ is defined by (3.38), then $s \in$ $[0,1)$, so that $v_{0} \in \dot{H}^{s}\left(\mathbb{R}^{N}\right),|\cdot| v_{0} \in \dot{H}^{s}\left(\mathbb{R}^{N}\right), \nabla v_{0} \in \dot{H}^{s}\left(\mathbb{R}^{N}\right)$. Therefore, it follows from Remark 3.2 that $e^{i t \Delta} v_{0} \in L^{a}\left((0,1), L^{\rho}\left(\mathbb{R}^{N}\right)\right),|\cdot| e^{i t \Delta} v_{0} \in L^{a}\left((0,1), L^{\rho}\left(\mathbb{R}^{N}\right)\right)$, $e^{i t \Delta} \nabla v_{0} \in L^{a}\left((0,1), L^{\rho}\left(\mathbb{R}^{N}\right)\right)$ and $\left\|e^{i t \Delta} \nabla v_{0}\right\|_{L^{a}\left((0,1), L^{\rho}\right)} \leq\left\|v_{0}\right\|_{H^{2}}$. Thus we see that if $\left\|v_{0}\right\|_{H^{2}}$ is sufficiently small, then $v_{0}$ satisfies the assumptions of Theorem 3.1. Let $v \in C([0,1], X)$ be the corresponding solution of (3.1). Following [4], we apply the pseudo-conformal transformation. More precisely, let the variables $(s, y) \in$ $[0, \infty) \times \mathbb{R}^{N}$ be defined by

$$
s=\frac{t}{1-t}, \quad y=\frac{x}{1-t}
$$

for $(t, x) \in[0,1) \times \mathbb{R}^{N}$. We define $u$ on $[0, \infty) \times \mathbb{R}^{N}$ by

$$
u(s, y)=(1-t)^{N / 2} e^{i \frac{|x|^{2}}{4(1-t)}} v(t, x) .
$$

It follows that $u \in C([0, \infty), X)$, and is a solution of $(1.2)$ on $[0, \infty)$. Finally, since $v \in C([0,1], X)$, it follows from Proposition 3.14 in [4] that there exists $u^{+} \in X$ such that $e^{-i s \Delta} u(s) \rightarrow u^{+}$in $X$ as $s \rightarrow \infty$. This completes the proof.

Remark 3.3. The conditions on the initial value $u_{0}$ in Theorem 1.2 are stronger than the conditions that are actually used in the proof. These latter conditions are expressed in terms of $v_{0}=e^{i \frac{|x|^{2}}{4}} u_{0}$ in formulas (3.8)-(3.11). Intermediate conditions are given in Remark 3.2.

\section{REFERENCES}

[1] Bergh J. and Löfström J. Interpolation spaces. An introduction. Grundlehren der Mathematischen Wissenschaften 223 . Springer-Verlag, Berlin-New York, 1976. (MR0482275) (doi: 10.1007/978-3-642-66451-9)

[2] Cazenave T. Semilinear Schrödinger equations, Courant Lecture Notes in Mathematics, 10. New York University, Courant Institute of Mathematical Sciences, New York; American Mathematical Society, Providence, RI, 2003. (MR2002047)

[3] Cazenave T. and Weissler F. B. The Cauchy problem for the critical nonlinear Schrödinger equation in $H^{s}$, Nonlinear Anal. 14 (1990), no. 10, 807-836. (MR1055532) (doi: $10.1016 / 0362-546 \mathrm{X}(90) 90023-\mathrm{A}$ ) 
[4] Cazenave T. and Weissler F. B. Rapidly decaying solutions of the nonlinear Schrödinger equation, Comm. Math. Phys. 147 (1992), 75-100. (MR1171761) (link: http://projecteuclid.org/euclid.cmp/1104250527)

[5] Deng K. and Levine H.A. The role of critical exponents in blow-up theorems: the sequel, J. Math. Anal. Appl. 243 (2000), no. 1, 85-126. (MR1742850) (doi: 10.1006/jmaa.1999.6663)

[6] Foschi D. Inhomogeneous Strichartz estimates, J. Hyperbolic Differ. Equ. 2 (2005), no. 1, 1-24. (MR2134950) (doi: 10.1142/S0219891605000361)

[7] Fujita H. On the blowing-up of solutions of the Cauchy problem for $u_{t}=\Delta u+u^{\alpha+1}$, J. Fac. Sci. Univ. Tokyo Sect. IA Math. 13 (1966), 109-124. (MR0214914) (link: http://repository.dl.itc.u-tokyo.ac.jp/dspace/bitstream/2261/6061/1/jfs130201.pdf)

[8] Ginibre J., Ozawa T. and Velo G. On the existence of the wave operators for a class of nonlinear Schrödinger equations, Ann. Inst. H. Poincaré Phys. Théor. 60 (1994), no. 2, 211-239. (MR1270296) (link: http://archive.numdam.org/article/AIHPA_1994__60_2_211_0.pdf)

[9] Ginibre J. and Velo G. Scattering theory in the energy space for a class of nonlinear Schrödinger equations, J. Math. Pures Appl. (9) 64 (1985), no. 4, 363-401. (Mr0839728)

[10] Kato T. On nonlinear Schrödinger equations, Ann. Inst. H. Poincaré Phys. Théor. 46 (1987), no. 1, 113-129. (MR0877998) (link: http://www .numdam.org/item?id=AIHPA_1987_-46_1_113_0)

[11] Kato T. An $L^{q, r}$-theory for nonlinear Schrödinger equations. In Spectral and Scattering Theory and Applications, 223-238, Adv. Stud. Pure Math., 23, Math. Soc. Japan, Tokyo, 1994. (MR1275405)

[12] Levine H.A. The role of critical exponents in blow-up theorems, SIAM Rev. 32 (1990), no. 2, 262-288. (MR1056055) (doi: 10.1137/1032046)

[13] Nakanishi K. and Ozawa T. Remarks on scattering for nonlinear Schrödinger equations, NoDEA Nonlinear Differential Equations Appl. 9 (2002), no. 1, 45-68. (MR1891695) (doi: $10.1007 / \mathrm{s} 00030-002-8118-9$ )

[14] Okazawa N. and Yokota T. Monotonicity method for the complex Ginzburg-Landau equation, including smoothing effect. Proceedings of the Third World Congress of Nonlinear Analysts, Part 1 (Catania, 2000). Nonlinear Anal. 47 (2001), no. 1, 79-88. (MR1970632) (doi: 10.1016/S0362-546X(01) 00158-4)

[15] Strauss W.A. Nonlinear scattering theory, in Scattering Theory in Mathematical Physics, NATO Advanced Study Institutes Series Volume 9, 53-78. Springer Netherlandsl, 1974. (doi: 10.1007/978-94-010-2147-0_3)

[16] Vilela M.-C. Inhomogeneous Strichartz estimates for the Schrödinger equation, Trans. Amer. Math. Soc. 359 (2007), no. 5, 2123-2136. (MR2276614) (doi: 10.1090/S0002-9947-06-04099-2)

${ }^{1}$ Université Pierre et Marie Curie \& CNRS, Laboratoire Jacques-Louis Lions, B.C. 187, 4 Place Jussieu, 75252 Paris Cedex 05, France

E-mail address: thierry.cazenave@upmc.fr

${ }^{2}$ Centro de Matemática e Aplicações Fundamentais, Universidade de Lisboa, Avenida Prof. Gama Pinto 2, 1649-003 Lisboa, Portugal

E-mail address: sfcorreia@fc.ul.pt

${ }^{3}$ Instituto de Matemática, Universidade Federal do Rio de Janeiro, Caixa Postal 68530, 21944-970 Rio dE JANEIRo, R.J., BRAZiL

E-mail address: flavio@labma.ufrj.br

${ }^{4}$ Université Paris 13, Sorbonne Paris Cité, CNRS UMr 7539 LAGA, 99 Avenue J.-B. Clément, F-93430 Villetaneuse, France

E-mail address: weissler@math.univ-paris13.fr 